\title{
Artificial Evolution Methods in the Biological and Biomedical Sciences
}

\author{
Jason H. Moore, ${ }^{1}$ Janet Clegg, ${ }^{2}$ Elena Marchiori, ${ }^{3}$ Marylyn Ritchie, ${ }^{4}$ and Stephen Smith ${ }^{2}$ \\ ${ }^{1}$ Department of Genetics, Dartmouth College, Hanover, NH 03755, USA \\ ${ }^{2}$ Department of Electronics, University of York, Heslington, York YO10 5DD, UK \\ ${ }^{3}$ Faculty of Science, Radboud University, P. O. Box 9102, 6500 HC Nijmegen, The Netherlands \\ ${ }^{4}$ Department of Molecular Physiology and Biophysics, Vanderbilt University, 2201 West End Avenue, Nashville, TN 37232, USA
}

Correspondence should be addressed to Jason H. Moore, jason.h.moore@dartmouth.edu

Received 29 July 2009; Accepted 29 July 2009

Copyright ( 92009 Jason H. Moore et al. This is an open access article distributed under the Creative Commons Attribution License, which permits unrestricted use, distribution, and reproduction in any medium, provided the original work is properly cited.

Artificial evolution has emerged as a powerful computational strategy for solving complex problems in the biological and biomedical sciences. Inspired by biological evolution, artificial evolution is attractive because it employs stochastic search algorithms that are inherently parallel. As such, these novel approaches are ideal for biological and biomedical problems that are high-dimensional, noisy, and very complex. The papers that appear in this special issue of the Journal of Artificial Evolution and Applications were rigorously peer reviewed and represent a wide range of different algorithms and application areas. As such, the papers in this special issue represent both the depth and breadth of artificial evolution and its potential applications. Papers in this volume cover a range of artificial evolution algorithms including, for example, genetic algorithms and genetic programming. Application areas span multiple different areas including the classification of microarray data, classification and diagnosis of cancer, multiple sequence alignment, ecological simulation, population genetics, and neurological discrimination.

The application of artificial evolution to problems in medicine is particularly exciting. The use of evolutionary algorithms such as genetic programming is becoming more common and the paper "Classification of oncologic data with genetic programming" by Leonardo Vannesci et al. is a particularly good example in which the critical fitness function employed is based on a clinical evaluation, in this case the area under the receiver operating characteristic curve and a measure of correctly classified instances. Senhua Yen and Dipankar Dasgupta describe the development of a novel algorithm inspired by the immune system in their paper "Conserved self pattern recognition algorithm with novel detection strategy applied to breast cancer diagnosis." The aim is to aid breast cancer diagnosis by analysing the cytological characteristics of breast fine needle aspirates using this novel pattern recognition approach. Although the methods and algorithms employed are very different, these papers demonstrate the potential impact of applying artificial evolution to the whole spectrum of medical applications.

The biological and biomedical sciences may be the ideal application areas for artificial evolution given the complexity of the data and the complexity of the processes and patterns being studied. This special issue provides a sampling of the state of the art in the field and should provide many novel ideas for readers to try with their own problem-solving efforts.

Jason H. Moore Janet Clegg Elena Marchiori Marylyn Ritchie Stephen Smith 